Emilmary Maya; Julio Juvenal Aldana Zavala; Josía Isea

\title{
Liderazgo Directivo y Educación de Calidad
}

\section{Leadership Management and Quality Education}

\author{
Emilmary Maya \\ emilmayap@hotmail.com \\ Liceo Nacional Manuela Lugo de Reyes \\ Julio Juvenal Aldana Zavala \\ julioaldanazavala@gmail.com \\ Universidad Nacional Experimental Francisco de Miranda \\ https://orcid.org/0000-0002-7934-9103 \\ Josía Isea \\ josiaisea@gmail.com \\ Universidad Nacional Experimental Francisco de Miranda \\ Grupo de Investigación CIENCIAMATRIA \\ https://orcid.org/0000-0001-8921-6446
}

Recibido: $17 / 10 / 2018$

Aprobado: 28/11/2018

\section{RESUMEN}

El objetivo del estudio es determinar la relación del liderazgo directivo en la consecución de una educación de calidad. La metodología se basó en un estudio correlacional transaccional, el diseño fue de campo no experimental. Entre los principales resultados se tiene que existe correlación positiva baja entre las variables de estudio, lo cual implica que el liderazgo directivo influye directamente en la consecución 
de una educación de calidad, es decir, que uno de los factores que hacen posible la denominada educación de calidad, es la presencia de un liderazgo directivo efectivo, asertivo, democrático, participativo, que involucre al personal docente en los planes de trabajo de modo activo y protagónico, cuando este estilo de liderazgo se hace presente, la organización camina hacia la calidad, cuando el director aplica otros estilos de liderazgo, la calidad en la gestión de una educación de calidad se deteriora y aleja de lo esperado en aras de conseguir una educación de calidad.

Palabras clave: Liderazgo, Calidad, Educación, Gestión, Proceso Enseñanza Aprendizaje.

\begin{abstract}
The objective of the study is to determine the relationship of the leadership in the achievement of a quality education. The methodology was based on a transactional correlational study, the design was non-experimental field. Among the main results, there is a low positive correlation between the study variables, which implies that directing leadership directly influences the achievement of a quality education, that is, one of the factors that make the so-called education possible. quality, is the presence of an effective, assertive, democratic, participative leadership leadership that involves the teaching staff in the work plans in an active and protagonistic way, when this style of leadership is present, the organization walks towards quality, when the director applies other styles of leadership, quality in the management of a quality education deteriorates and away from what was expected in order to achieve a quality education.
\end{abstract}

Key words: Leadership, Quality, Education, Management, Teaching - Learning Process.

\title{
INTRODUCCIÓN
}

La educación es una de las dimensiones fundamentales de la reforma del Estado Venezolano, tiene como finalidad la formación integral del individuo para que asuma las transformaciones que demanda la sociedad del conocimiento, mediante el cual, los estudiantes se formar para aprender a lo largo de la vida, con base en la investigación y en valores democráticos de participación protagónica, los cuales son necesarios para 


\section{CIENCIAMATRIA \\ Revista Interdisciplinaria de Humanidades, Educación, Ciencia y Tecnología \\ Año V. Vol. V. №9. Julio - Diciembre 2019 \\ Hecho el depósito de ley: pp201602FA4721 \\ ISSN-L: 2542-3029; ISSN: 2610-802X \\ Universidad Nacional Experimental Francisco de Miranda (UNEFM). Santa Ana de Coro. Venezuela}

Emilmary Maya; Julio Juvenal Aldana Zavala; Josía Isea

avanzar en el proceso de cambio que se está dando en los actuales momentos en el mundo educativo. De esta manera, se promueve a la persona como actor y autor de su historia educativa - social, lo cual se sustenta desde la equidad, libertad, participación, cooperación y solidaridad como fundamentos para su formación integral.

A este respecto, el (Ministerio del Poder Popular para la Educación, 2007) señala que “... el rol que cumple la escuela como eje del proceso educativo resulta de gran relevancia, debido a que dentro de su estructura organizativa, el primer nivel lo lideriza el gerente educativo, quien tiene en sus manos elementos indispensables" (p.65). Para promover las transformaciones desde la escuela, se requiere la existencia de un grupo de colaboradores, un alumnado en formación, el liderazgo que la profesión docente en sí misma incluye, una comunidad de padres o representantes y un espacio físico constituido por la escuela como expresión concreta y tangible de la política educativa de un país.

El líder directivo educativo debe poseer una serie de rasgos para poder desempeñar un buen papel, debe ser motivador, guía y mediador en situaciones, conflictos, dentro del centro escolar, buscando la satisfacción de los empleados y el bienestar de la misma. En tal sentido, el liderazgo del director juega un papel importante en la dirección de la organización educativa, lo cual permite generar una vinculación entre el liderazgo y la calidad de los procesos desarrollados en la institución, como en la satisfacción de los profesores y toda la comunidad escolar.

En otras palabras, para que una institución sea de calidad debe existir el apoyo mancomunado entre el personal del centro escolar (coordinadores, docentes, secretarias, obreros, estudiantes, padres y representantes) y la comunidad en general, siendo el director el impulsador, motivador e interesado porque las metas se cumplan. En este sentido, el director de la organización educativa, se constituye en un elemento primordial para el buen desarrollo de los procesos educativos, debido que la calidad de su gestión puede determinar el nivel de desempeño de la comunidad escolar en 


\section{CIENCIAMATRIA \\ Revista Interdisciplinaria de Humanidades, Educación, Ciencia y Tecnología \\ Año V. Vol. V. №9. Julio - Diciembre 2019 \\ Hecho el depósito de ley: pp201602FA4721 \\ ISSN-L: 2542-3029; ISSN: 2610-802X \\ Universidad Nacional Experimental Francisco de Miranda (UNEFM). Santa Ana de Coro. Venezuela}

Emilmary Maya; Julio Juvenal Aldana Zavala; Josía Isea

general.

El director debe garantizar la enseñanza y el aprendizaje a sus estudiantes, apoyar a su personal, así como hacer que la institución sea siempre reconocida por la calidad educativa que desarrolla en beneficio de la sociedad. Es importante destacar que es importante que la gestión del director educativo, no se vea limitada por la burocracia presente actualmente en los centros escolares. Como primera responsabilidad del establecimiento educacional, es preciso garantizar el éxito educativo a todo estudiante, pero esto no puede quedar enteramente al arbitrio de lo que cada profesor, con mayor o menor suerte, haga en su aula.

Por tanto es necesaria que la calidad de la enseñanza y del aprendizaje que ofrece la institución educativa, sea generada como resultado de una gestión directiva que promueva el progreso de la organización en su contexto general. Así lo manifiestan (Stoll y Temperley, 2009) cuando sostienen que

“... los líderes escolares sólo pueden influir en los resultados de los estudiantes si cuentan con autonomía suficiente para tomar decisiones importantes acerca del currículum y la selección y formación de maestros; además, sus principales áreas de responsabilidad deberán concentrarse en mejorar el aprendizaje de los estudiantes. Los países optan cada vez más por la toma de decisiones descentralizadas y por equilibrar éstas con una mayor centralización de los regímenes de rendición de cuentas, tal como las pruebas estandarizadas. (p. 13).

Según los planteamientos expuestos, el objetivo de cada equipo directivo, es diseñar, hacer seguimiento y mejorar los procesos críticos que añaden valor a la organización, que permitan conseguir resultados de calidad. Actualmente tiene importancia hacer énfasis en el liderazgo directivo por cuanto las organizaciones y el sistema educativo específicamente necesitan ser conducidos por personas que tengan capacidad de dominio de grupos; debido a que ello permite poder afrontar los cambios, desafíos, que trae el avance de la ciencia y tecnología. En los centros educativos, la figura del director y su papel de líder es importante para lograr que la organización trabaje correctamente 


\section{CIENCIAMATRIA \\ Revista Interdisciplinaria de Humanidades, Educación, Ciencia y Tecnología \\ Año V. Vol. V. No9. Julio - Diciembre 2019 \\ Hecho el depósito de ley: pp201602FA4721 \\ ISSN-L: 2542-3029; ISSN: 2610-802X \\ Universidad Nacional Experimental Francisco de Miranda (UNEFM). Santa Ana de Coro. Venezuela}

Emilmary Maya; Julio Juvenal Aldana Zavala; Josía Isea

y se logren los objetivos previstos por la institución.

Se hace necesario abordar el gran valor que ocupa esta habilidad humana en el campo gerencial educativo. Ciertamente, se afirma que en esta habilidad algunos gerentes logran la socialización e integración en sus subalternos. Sin embargo, (Weinstein, 2009) manifiesta "... para nadie es un secreto, que la sobrecarga de actividades burocráticas y administrativas impide el ejercicio de un liderazgo pedagógico" (p.11).

Por consiguiente, es pertinente investigar sobre los aspectos relacionados al liderazgo educativo y a la educación de calidad como producto de una gestión directiva fomentada en pro del crecimiento integral de la organización. Dentro de este marco ha de considerarse la relevancia de esta investigación en el Liceo Nacional "Manuela Lugo de Reyes" del municipio Tocópero del estado Falcón, institución adscrita al Municipio Escolar Integral Tocópero (M.E.I.T.), por cuanto desde esta perspectiva se deja en claro que en las instituciones educativas del estado Falcón y en el mencionado municipio se presentan problemas en la toma de decisiones del personal directivo (liderazgo directivo), lo cual conlleva a gestiones para una educación de calidad o no. Del planteamiento formulado anteriormente, se deriva la siguiente interrogante:

¿Cuál es la relación del liderazgo directivo en la consecución de una educación de calidad?

\section{Objetivo de la investigación}

Determinar la relación del liderazgo directivo en la consecución de una educación de calidad.

\section{REFERENCIAL TEÓRICO}

\section{Liderazgo Directivo}

En función de describir la variables liderazgo directivo, se presenta a (Farre y Lasheras, 2002) quienes mencionan que el liderazgo directivo 


\section{CIENCIAMATRIA \\ Revista Interdisciplinaria de Humanidades, Educación, Ciencia y Tecnología \\ Año V. Vol. V. №9. Julio - Diciembre 2019 \\ Hecho el depósito de ley: pp201602FA4721 \\ ISSN-L: 2542-3029; ISSN: 2610-802X \\ Universidad Nacional Experimental Francisco de Miranda (UNEFM). Santa Ana de Coro. Venezuela}

Emilmary Maya; Julio Juvenal Aldana Zavala; Josía Isea

Es la tarea distinguida por la fijación de normas, la organización del trabajo y la concentración en las metas, es muy eficaz para mantener a un grupo centrado en una misión. El líder directivo es aquel que tiene capacidad suficiente para impartir órdenes apropiadas. (p. 125).

En el contexto educativo la función del director o directivo se centra mayormente en la dirección de la institución y el liderazgo curricular. El liderazgo del director es un factor determinante en la mejora de lo que acontece en los planteles educativos y un factor que incide en el desarrollo de la institución y el aprendizaje de los estudiantes.

Ejercer liderazgo es un gran reto para el director de escuelas. Es una gestión de responsabilidad que busca la transformación de la institución en una organización ágil y dinámica que logre los resultados esperados de acuerdo con los estándares de calidad de la institución y el sistema educativo en general. Dentro de las características del director como líder de una comunidad escolar, (Sergiovanni 2001, p. 47) formula cinco estilos de liderazgo:

1. Líder técnico: planifica, organiza, coordina, desarrollo y establece estructuras organizativas para asegurar una eficacia óptima en la institución.

2. Líder humanista: le otorga mayor importancia a las relaciones humanas, a la competencia interpersonal y a las técnicas de motivación instrumental. Apoya, anima y proporciona oportunidades de desarrollo profesional a los miembros de la institución.

3. Líder educativo: desarrolla programas educativos y de supervisión clínica, diagnostica problemas educativos, orienta a los profesores, promueve la supervisión, la evaluación y se preocupa por el desarrollo del currículo.

4. Líder simbólico: asume el papel de jefe y pone atención en la selección de metas y comportamientos importantes para el plantel. Recorre la institución, visita los salones, mantiene contacto directo y permanente con los estudiantes, establece prioridades en los intereses educativos, preside ceremonia y actividades oficiales y transmite visión unificadora a través de las palabras y las acciones. 


\section{CIENCIAMATRIA \\ Revista Interdisciplinaria de Humanidades, Educación, Ciencia y Tecnología \\ Año V. Vol. V. №9. Julio - Diciembre 2019 \\ Hecho el depósito de ley: pp201602FA4721 \\ ISSN-L: 2542-3029; ISSN: 2610-802X \\ Universidad Nacional Experimental Francisco de Miranda (UNEFM). Santa Ana de Coro. Venezuela}

Emilmary Maya; Julio Juvenal Aldana Zavala; Josía Isea

5. Líder cultural: define, fortalece y articula los valores, las creencias y raíces culturales que dan a la escuela su identidad única. Articula la misión de la institución, mantiene tradiciones, socializa a los miembros de la comunidad escolar a la cultura de la escuela y desarrolla y manifiesta un sistema de símbolos a lo largo del tiempo que refleje la cultura escolar. Vincula y promueve la creencia de estudiantes, profesores, padres y demás miembros de la comunidad escolar en el trabajo de la institución.

Las características descritas, son importantes tenerlas en consideración, por cuanto permiten articular la posibilidad de proyectar un liderazgo directivo cohesionado a diversas aristas que promuevan el trabajo sinérgico en la organización desde una perspectiva integral y holística, dando paso a diversos estilos de liderazgos educativos, pero que en el fondo convergen en la generación de una educación de calidad.

El desarrollar un estilo de liderazgo significa que la persona que dirige y lidera una organización va mostrar una serie de conductas y comportamientos comunes para con los miembros de la organización. Pero no siempre la persona puede mostrar un solo estilo de liderazgo ya que hay situaciones o contexto donde amerita y es necesario cambiar de estilo.

Por consiguiente, (Lussier, 2002) afirma que el estilo de liderazgo "es la combinación de rasgos, destrezas y comportamientos a los que recurren los líderes al interactuar con los seguidores" (p. 67). Aunque un estilo de liderazgo se compone de rasgos y destrezas, el elemento fundamental es el comportamiento, puesto que es el patrón de conducta relativamente constante que caracteriza al líder. Por otro lado, (Rojas, 1997) destaca que a pesar de que "algunos sujetos tengan cargos similares en un grupo, se diferencian sustancialmente unos de otros por varios factores en especial por la personalidad de cada líder, lo cual, lo hace único en su género" (p. 104).

Particularmente se hace evidente en lo relativo al tipo de estilo en las relaciones con los subordinados. Estas es la explicación fundamental de la existencia de los líderes autoritarios, pusilánimes, democráticos y situacional, que son las formas más 


\section{CIENCIAMATRIA \\ Revista Interdisciplinaria de Humanidades, Educación, Ciencia y Tecnología \\ Año V. Vol. V. №9. Julio - Diciembre 2019 \\ Hecho el depósito de ley: pp201602FA4721 \\ ISSN-L: 2542-3029; ISSN: 2610-802X \\ Universidad Nacional Experimental Francisco de Miranda (UNEFM). Santa Ana de Coro. Venezuela}

Emilmary Maya; Julio Juvenal Aldana Zavala; Josía Isea

destacadas en el ejercicio del liderazgo dentro de una organización. En base a esta divergencia es que existen diferentes estilos que aplica el líder en el ejercicio de sus funciones por ello podemos encontrar en las organizaciones lideres autoritario, pusilánimes o laissez faire, democrático o situacional.

En este sentido el liderazgo autoritario o autocrático según diversos autores coinciden en plantear que este estilo de liderazgo es aquel donde todo el poder y la autoridad se centran en una sola persona, es quien toma las decisiones, los subordinados son muy dependientes y la estructura organizativa es rígida. Los líderes son controladores y los subordinados asumen actitudes de sumisión o rebeldía.

Por otro lado, (Álvarez, 2001), plantea, "Toda la dinámica del grupo se estructura desde el líder que fija los objetivos y decide los recursos a aplicar" (p. 20). El líder así concebido centraliza totalmente la autoridad y las decisiones. Los subordinados no tienen ninguna libertad para elegir. El líder autocrático es dominante, imparte órdenes y espera obediencia plena y ciega de los subordinados. Los grupos sometidos a un líder autocrático presentan el mayor volumen de trabajo productivo y evidente señales de tensión, frustración y agresividad. El líder autocrático es temido por el grupo, que sólo trabaja cuando aquél está presente y enfatiza todo en el líder, se basa en amenaza y castigo.

Pues bien, en lo referente a las organizaciones educativas, donde el docente día a día se enfrenta a situaciones dinámicas, activas que no es convenientes que el directivo centralice toda la autoridad, sin embargo, algunos directores de las instituciones de educación básica, asumen este estilo y le da resultados óptimos, con respecto al control fiscalizador del personal docente. No obstante pudiera con esto dejar de desarrollar por parte del directivo la autonomía y la creatividad ejercida por los docentes, elemento esencial dentro de los diversos roles desempeñados por los educadores.

Por otro lado, está el estilo democrático o participativo consulta y persuade a los subordinados y alienta la participación. Es el estilo más recomendable en las acciones 


\section{CIENCIAMATRIA \\ Revista Interdisciplinaria de Humanidades, Educación, Ciencia y Tecnología \\ Año V. Vol. V. №9. Julio - Diciembre 2019 \\ Hecho el depósito de ley: pp201602FA4721 \\ ISSN-L: 2542-3029; ISSN: 2610-802X \\ Universidad Nacional Experimental Francisco de Miranda (UNEFM). Santa Ana de Coro. Venezuela}

Emilmary Maya; Julio Juvenal Aldana Zavala; Josía Isea

pedagógicas y administrativas de las organizaciones educativas. La relación es democrática y el líder se sitúa como uno más dentro del grupo. Crea relaciones de amistad, confianza y dialogo. Permite que los integrantes se expresen libremente. Las actividades se hacen según los deseos y posibilidades de los integrantes.

\section{Calidad Educativa}

En este sentido, (Calero, 1998) señala que "el trabajo del director para el mejoramiento de la educación es la toma adecuada de decisiones" (p. 215). Decidir en forma acertada es un proceso tan importante como el proceso mismo, con esto quiere decir que para encontrar una excelencia educativa se requiere de múltiples factores: entre otros, de profesores efectivos, de condiciones pedagógicas buenas, de estudiantes en buenas condiciones físicas y psicológicas, de políticas educativas claras, de universos valorativos identificables, de fluidez financiera y administrativa a nivel macro y también de la existencia cotidiana de las decisiones que se toman. Las grandes políticas y los grandes planes se convierten en decisiones reales y hechos visibles, a través de sus decisiones.

Un director es un educador no porque está involucrado directamente en el hecho pedagógico, sino porque a través de sus decisiones puede contribuir a hacerlo más efectivo, facilitándole al docente su tarea, garantizándole el funcionamiento efectivo de la organización y/o dándole sentido dentro del entorno social. El mismo autor (Calero, 2006, p. 280), puntualiza que ser director implica decidir en función de:

1. Crear condiciones favorables al hecho pedagógico.

2. Garantizar el funcionamiento eficiente de la administración.

3. Influir y negociar con el medio social los procesos, insumos e intereses que requiere una institución para avanzar hacia mejores niveles.

Aspectos fundamentales que deben ser tenidos en cuenta a la hora de fomentar una organización educativa basada en la calidad, eficiencia, sinergia, con miras a trabajar 


\section{CIENCIAMATRIA \\ Revista Interdisciplinaria de Humanidades, Educación, Ciencia y Tecnología \\ Año V. Vol. V. №9. Julio - Diciembre 2019 \\ Hecho el depósito de ley: pp201602FA4721 \\ ISSN-L: 2542-3029; ISSN: 2610-802X \\ Universidad Nacional Experimental Francisco de Miranda (UNEFM). Santa Ana de Coro. Venezuela}

Emilmary Maya; Julio Juvenal Aldana Zavala; Josía Isea

sistemáticamente desde el trabajo en equipo, no solo al cumplimiento de metas y objetivos, sino, en procura del crecimiento integral de las partes.

Para conseguir calidad en la educación se debe evitar la segmentación, y por el contrario, potenciar el rol de integración de la educación en un contexto desigual que busque desarrollar un proyecto educativo para la transformación social. Así mismo el autor afirma que, cuando se asegura la participación de los actores locales en el proceso educativo, se asegura la calidad.

En este sentido las posibilidades de mejora de las instituciones escolares, se basan en reconocer que estos procesos no pueden sólo prescribirse, por cuanto en ellos inciden factores tales como el compromiso, la iniciativa y la implicación, propios del contexto de cada institución, y que el sistema educativo debe posibilitar condiciones y contextos para que los cambios puedan tener lugar. Esto implica poner en tensión los factores que pueden generar un cambio, recuperando los procesos y relaciones que favorezcan la emergencia de dinámicas autónomas, devolviendo el protagonismo a los actores y que - por ello mismo - puedan tener mayor grado de permanencia.

\section{METODOLOGÍA DE LA INVESTIGACIÓN}

La investigación se enmarcó en los criterios de un estudio correlacional transaccional, el cual tiene como objetivo indagar la incidencia y los valores en que se manifiesta una o más variables. El procedimiento consiste en medir en un grupo de personas u objetos una o más variables y proporcionar su descripción. Al respecto (Chávez, 2007) afirma que estos estudios correlaciónales tienen "como propósito determinar el grado de relación entre variables, detectando hasta qué punto las alteraciones de una, dependen de la otra, el cual da por resultado un coeficiente r" (p. 137).

El diseño asumido para abordar las respuestas al problema planteado en la investigación es de campo, por cuanto se recopiló la información directamente donde se producían los hechos, por lo cual (Sabino, 2002), manifiesta “... los diseños de campo son los que se refiere a los métodos a emplear cuando los datos de interés se recogen 


\section{CIENCIAMATRIA \\ Revista Interdisciplinaria de Humanidades, Educación, Ciencia y Tecnología \\ Año V. Vol. V. №9. Julio - Diciembre 2019 \\ Hecho el depósito de ley: pp201602FA4721 \\ ISSN-L: 2542-3029; ISSN: 2610-802X \\ Universidad Nacional Experimental Francisco de Miranda (UNEFM). Santa Ana de Coro. Venezuela}

Emilmary Maya; Julio Juvenal Aldana Zavala; Josía Isea

de manera directa de la realidad mediante el trabajo concreto del investigador y su equipo". (p.89).

Así mismo es una investigación no experimental, por cuanto se observaron los fenómenos a estudiar tal y como se dieron en su contexto natural, para luego ser analizados, tal como lo afirma (Hernández, Fernández y Baptista, 2009): “... la investigación de tipo no experimental se realiza sin manipular deliberadamente las variables, los fenómenos se observan tal y como se dan en su contexto natural, para después ser analizados". (p. 81).

En cuanto a la muestra de estudio se trabajó con el personal docente del Liceo Nacional "Manuela Lugo de Reyes" del municipio Tocópero del estado Falcón, puesto que se consideró necesario tomarla en su totalidad, debido que estadísticamente era manejable a los efectos de la aplicación de los instrumentos de recolección de información. Según (Arias, 2004) “... las poblaciones pequeñas deben tomarse en su totalidad como una forma de garantizar la consecución de datos confiables" (p. 26), en este sentido la población estuvo conformada por una (01) sub-directora, una (01) coordinadora y diez (10) profesores.

Para la recolección de datos se empleó como técnica la observación directa y la encuesta. El instrumento aplicado en la recolección de datos fue el cuestionario conformado con 36 ítems y dos alternativas de respuesta (SI - NO) para cada uno. Los primeros 18 ítems miden la opinión de los encuestados con relación a la variable Liderazgo Educativo y los restantes 18, la variable Calidad Educativa. Todo esto posibilitó el análisis, procesamiento y descripción de los resultados obtenidos en el desarrollo de la investigación.

Así mismo, para el cálculo de la correlación se usó el coeficiente de correlación de Pearson, el cual según (Hernández, Fernández y Baptista, 2009) es una prueba estadística para analizar la relación entre dos variables medidas por intervalos o por razón, el cual es conveniente para tal estudio. 


\section{ANÁLISIS DE LOS RESULTADOS}

La correlación permite hacer estimaciones del valor de una de ellas conociendo el valor de la otra variable. Los coeficientes de correlación son medidas que indican la situación relativa de los mismos sucesos respecto a las dos variables, es decir, son la expresión numérica que nos indica el grado de relación existente entre las 2 variables y en qué medida se relacionan. Son números que varían entre los límites +1 y -1 . Su magnitud indica el grado de asociación entre las variables; el valor $r=0$ indica que no existe relación entre las variables; los valores son indicadores de una correlación perfecta positiva (al crecer o decrecer $X$, crece o decrece $Y$ ) o negativa (Al crecer o decrecer $X$, decrece o crece Y). Así mismo, para interpretar el coeficiente de correlación se utilizó la siguiente escala:

\section{Cuadro 1.}

Valor de las correlaciones

\begin{tabular}{ll}
\hline Valor & Significado \\
\hline-1 & Correlación negativa grande y \\
perfecta & Correlación negativa muy alta \\
$-0,9$ a $-0,99$ & Correlación negativa alta \\
$-0,7$ a $-0,89$ & Correlación negativa moderada \\
$-0,4$ a $-0,69$ & Correlación negativa baja \\
$-0,2$ a $-0,39$ & Correlación negativa muy baja \\
$-0,01$ a $-0,19$ & Correlación nula \\
0 & Correlación positiva muy baja \\
0,01 a 0,19 & Correlación positiva baja \\
0,2 a 0,39 & Correlación positiva moderada \\
0,4 a 0,69 & Correlación positiva alta \\
0,7 a 0,89 & Correlación positiva muy alta \\
0,9 a 0,99 & Correlación positiva grande y \\
1 & perfecta \\
\hline
\end{tabular}

Fuente: Suárez (2010) 


\section{CIENCIAMATRIA \\ Revista Interdisciplinaria de Humanidades, Educación, Ciencia y Tecnología \\ Año V. Vol. V. №9. Julio - Diciembre 2019 \\ Hecho el depósito de ley: pp201602FA4721 \\ ISSN-L: 2542-3029; ISSN: 2610-802X \\ Universidad Nacional Experimental Francisco de Miranda (UNEFM). Santa Ana de Coro. Venezuela}

Emilmary Maya; Julio Juvenal Aldana Zavala; Josía Isea

Valor obtenido de la correlación entre las variables de estudio, aplicándose el software SPSS 21:

\section{0,001073636}

Rango de la correlación obtenida se ubica entre 0,01 a 0,19 , catalogándose como correlación positiva muy baja.

Con este resultado la calidad educativa se incrementará en la medida que el liderazgo educativo aumenta eficientemente y viceversa, es decir que ambas variables interactúan en favor de la otra; situación que puede generarse en la medida que el cuerpo directivo tenga actitudes asertivas, sinérgicas, hacia el liderazgo que ejercen en sus subalternos y supervisados.

\section{CONCLUSIONES}

Existe correlación positiva baja entre las variables de estudio, lo cual implica que el liderazgo directivo influye directamente en la consecución de una educación de calidad, es decir, que uno de los factores que hacen posible la denominada educación de calidad, es la presencia de un liderazgo directivo efectivo, asertivo, democrático, participativo, que involucre al personal docente en los planes de trabajo de modo activo y protagónico, cuando este estilo de liderazgo se hace presente, la organización camina hacia la calidad, cuando el director aplica otros estilos de liderazgo, la calidad en la gestión de una educación de calidad se deteriora y aleja de lo esperado en aras de conseguir una educación de calidad, por consiguiente, es pertinente tener en consideración lo siguiente:

- La institución se encuentra en una crisis de gestión y liderazgo institucional, debilidad que se puede convertir en una oportunidad de cambio y mejoramiento continuo de la organización.

- La falencia en la gestión educativa da la pauta para la implementación de cambios radicales. 


\section{CIENCIAMATRIA \\ Revista Interdisciplinaria de Humanidades, Educación, Ciencia y Tecnología \\ Año V. Vol. V. №9. Julio - Diciembre 2019 \\ Hecho el depósito de ley: pp201602FA4721 \\ ISSN-L: 2542-3029; ISSN: 2610-802X \\ Universidad Nacional Experimental Francisco de Miranda (UNEFM). Santa Ana de Coro. Venezuela}

Emilmary Maya; Julio Juvenal Aldana Zavala; Josía Isea

- La actual condición del cuerpo directivo no permite el desarrollo apropiado de una gestión y liderazgo dentro y fuera de la institución.

- La capacidad de gestión de liceo es muy débil para el desarrollo de proyectos de planificación en la institución educativa.

- No existe un modelo de gestión para el régimen de estudios en la institución que contribuya a su mejoramiento en el nivel de calidad educativa y que pueda generar un sistema de mejoramiento continuo.

- La capacitación y actualización del personal docente no es continua y permanente, provocando la falta de comunicación entre docentes y directivos.

- El bajo nivel de liderazgo en la institución no permite llegar a consensos en el proceso de enseñanza - aprendizaje.

- Los programas de estudios carecen de un grado de flexibilidad, para poder ser revisados, evaluados y retroalimentados.

- La institución no cuenta con una visión particular de llegar a ser una organización líder en calidad educativa.

- En la actualidad, el proceso de evaluación interna no se aprovecha como herramienta de mejora continua de la institución.

- La estructura piramidal, no sólo de esta institución sino de casi todas en el ámbito nacional concibe a las personas como un recurso y no como seres de creatividad, limitando de esta manera la óptima gestión y liderazgo en la institución.

En relación con lo anteriormente presentado, se puede considerar el papel de la Inteligencia Emocional como una herramienta que permita el desarrollo de liderazgos directivos pertinentes que impulsen la calidad educativa. Al respecto señalan Gómez y Salas de González. (2018):

En este contexto (Educativo), la inteligencia emocional representa un papel significativo, por lo tanto, resulta necesario que se desarrollen perfiles gerenciales basados en esta teoría, identificando fortalezas y debilidades a 


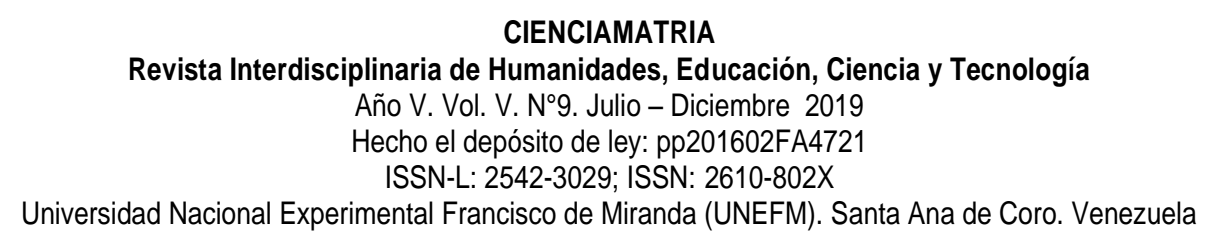

Emilmary Maya; Julio Juvenal Aldana Zavala; Josía Isea

nivel emocional, así como la aplicación de un cociente emocional que permita hoy más que nunca; el desarrollo de líderes innovadores en las escuelas, por cuanto estos se enfrentan a la necesidad de encontrar el equilibrio entre el currículum común que impone el sistema educativo y las tensiones específicas de cada escuela; entre el logro de los objetivos y un entorno saludable, entre la gestión de la diversidad en la escuela, la gestión participativa y la gestión comunitaria. (p. 25)

\section{REFERENCIAS CONSULTADAS}

1. Álvarez, M (2001) El liderazgo de la calidad total. Editorial Praxis S. A., Barcelona.

2. Arias, F. (2004). El proyecto de investigación. Guía para su elaboración. (3era ed.). Caracas, Venezuela: Editorial Episteme. Orial Ediciones.

3. Calero M (2006). Gestión Educativa. Lima: Abedul.

4. Calero M. (1998). Hacia la excelencia de la educación. Lima: San Marcos.

5. Chávez, N. (2007). Introducción a la investigación educativa. Educativa. Ediciones Grafica, C.A. Maracaibo - Venezuela. 4ta Edición.

6. Farre Josep y lasheras Gracia (2002). Enciclopedia de la psicología. Barcelona: Océano.

7. Gómez, M., \& Salas de González, M. (2018). Inteligencia Emocional del Gerente Educativo y Clima Laboral en Escuelas Bolivarianas Venezolanas. Revista Arbitrada Interdisciplinaria Koinonía, 3(5), 10-26. Recuperado de http://fundacionkoinonia.com.ve/ojs/index.php/revistakoinonia/article/view/111/93

8. Hernández, R, Fernández, C, Baptista, P (2009) Metodología de la Investigación. Sexta edición. $M^{C}$ Graw Hill. México.

9. Lussier, R (2002). Liderazgo. México DF: Thomson.

10. Ministerio del Poder Popular para la Educación (2007) Calidad de la Educación Bolivariana en el Subsistema de Educación Media General. Ministerio del Poder Popular para la Educación. Caracas. 
11. Rojas, G (1997). Psicología social y nuevo líder. 1era edición. Santa Fe de Bogotá.

12. Sabino, C. (2002). Metodología Investigativa en Educación. Editorial Co - Bo. Caracas. Venezuela

13. Sergiovanni, T. (2001). Leadership. What's in for schools? Londres: Routledge Falmer.

14. Stoll, G y Temperley W (2009) El Líder en la Gerencia Educativa. Universidad Central de Venezuela. Rev. Ped v. 23 n. 68. Caracas.

15. Suárez, M. (2010). Correlación y regresión. Recuperado de: http://www.docentesinnovadores.net/Archivos/5862/CORRELACI\%C3\%93N\%20 Y\%20REGRESI\%C3\%93N\%20EMPLEANDO\%20EXCEL\%20Y\%20GRAPH.pdf

16. Weinstein, (2009) La evaluación educativa, su práctica y otras metáforas. Bogotá, Colombia. 